\title{
Optimization of Surface Display of DENV2 E Protein on a Nanoparticle to Induce Virus Specific Neutralizing Antibody Responses
}

\author{
Jason E. Coffman, ${ }^{\dagger}$ Stefan W. Metz, ${ }^{\ddagger}$ Alex Brackbill, ${ }^{\S}$ Molly Paul, ${ }^{\|}$Michael J. Miley, ${ }^{\S}$ \\ Joseph DeSimone, ${ }^{\dagger, \|, \perp}$ J. Christopher Luft, ${ }^{\#}$ Aravinda de Silva, ${ }^{\ddagger}$ and Shaomin Tian ${ }^{*}$, \\ ${ }^{\dagger}$ Department of Chemical and Biomolecular Engineering, North Carolina State University, Raleigh, North Carolina 27607, United \\ States \\ ${ }^{\ddagger}$ Department of Microbiology and Immunology, ${ }^{\S}$ Department of Pharmacology, ${ }^{\prime \prime}$ Department of Chemistry, ${ }^{\perp}$ Lineberger \\ Comprehensive Center, and "Eshelman School of Pharmacy, University of North Carolina, Chapel Hill, North Carolina 27599, \\ United States
}

\begin{abstract}
The dengue virus (DENV) causes over 350 million infections, resulting in $\sim 25,000$ deaths per year globally. An effective dengue vaccine requires generation of strong and balanced neutralizing antibodies against all four antigenically distinct serotypes of DENV. The leading liveattenuated tetravalent dengue virus vaccine platform has shown partial efficacy, with an unbalanced response across the four serotypes in clinical trials. DENV subunit vaccine platforms are being developed because they provide a strong safety profile and are expected to avoid the unbalanced immunization issues associated with live multivalent vaccines. Subunit vaccines often lack immunogenicity, requiring either a particulate or adjuvanted formulation. Particulate formulations adsorbing monomeric DENV-E antigen to the particle surface incite a strong immune response, but have no control of antigen presentation. Highly neutralizing epitopes are displayed by DENV-E quaternary structures. To control the display of DENV-E and produce quaternary structures, particulate formulations that covalently attach DENV-E to the particle surface are needed. Here we develop a surface attached DENV2-E particulate formulation, as well as analysis tools, using PEG hydrogel nanoparticles created with particle replication in nonwetting templates (PRINT) technology. We found that adding Tween-20 to the conjugation buffer controls DENV-E adsorption to the particle surface during conjugation, improving both protein stability and epitope display. Immunizations with the anionic but not the cationic DENV2-E conjugated particles were able to produce DENV-specific and virus neutralizing antibody in mice. This work optimized the display of DENV-E conjugated to the surface of a nanoparticle through EDC/NHS chemistry, establishing a platform that can be expanded upon in future work to fully control the display of DENV-E.
\end{abstract}

\section{INTRODUCTION}

Dengue virus (DENV) causes over 350 million infections worldwide each year and is endemic in tropical and subtropical regions resulting in nearly 4 billion people at risk of infection. ${ }^{1,2}$ The majority of infections are clinically asymptomatic, however, of the 100 million symptomatic cases per year, 1 million result in severe and even fatal disease such as dengue hemorrhagic fever or dengue shock syndrome. ${ }^{3}$ DENV is transmitted by mosquitos of the Aedes genus, which due to changes in climate, global travel, and urbanization are inhabiting more temperate regions, expanding the population at risk for infection. ${ }^{4-6}$ DENV can be divided into 4 antigenically distinct serotypes and severe disease has been linked to subsequent heterotypic infections. Primary infections generally cause mild to moderate symptoms and result in a long-term immunity to the infecting serotype. Secondary infections however often cause more severe and potentially fatal symptoms though a process called antibody dependent enhancement (ADE). ${ }^{7,8} \mathrm{ADE}$ occurs because cross-reactive antibodies induced by a primary infection enhance cell uptake, infectivity, and viremia titers of a heterotypic DENV serotype during secondary infections., ${ }^{9,10}$ Vaccination is believed to be the most effective means of protecting the public against DENV infection. Vaccine development is challenging because candidates must generate a balanced and neutralizing immune response to all four dengue serotypes to avoid the effects of $\mathrm{ADE}$ and be fully protective.

Several dengue vaccine platforms are currently in preclinical and clinical development. The first commercially available

Received: February 6, 2018

Revised: April 3, 2018

Published: April 27, 2018 
Scheme 1. Scheme of EDC/NHS Conjugation Reaction ${ }^{a}$

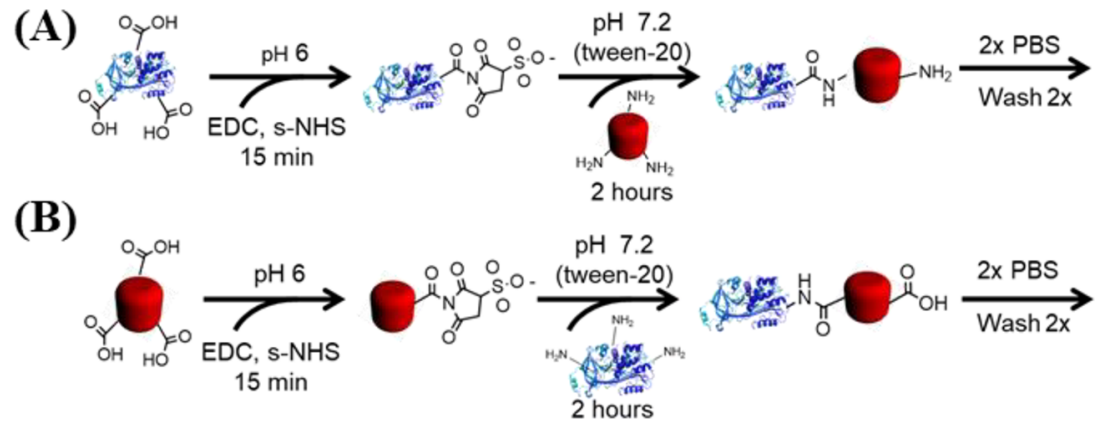

${ }^{a}$ (A) Protein is conjugated to cationic nanoparticles by activating the protein's carboxyl groups with EDC/s-NHS in pH 6 MES buffer, followed by adding the particle in $\mathrm{pH} 7.2$ sodium phosphate buffer $(0.1 \%$ Tween-20 v/v), allowing reaction to the particulate amine. (B) Protein is conjugated to anionic nanoparticles by activating the particle's carboxyl groups with EDC/s-NHS in pH 6 MES buffer, followed by adding the protein in pH 7.2 sodium phosphate buffer $(0.1 \%$ Tween- $20 \mathrm{v} / \mathrm{v})$, allowing reaction to protein amines.

dengue vaccine Dengvaxia (CYD-TDV), a live-attenuated dengue virus vaccine, was first licensed in December 2015, and is currently licensed by more than 10 endemic countries. $^{11,12}$ The live-attenuated virus based vaccine has shown a moderate pooled vaccine efficacy of $59.2 \%$ for all serotypes combined and only $43.0 \%$ against DENV $2 .^{13}$ Additionally, severe health risks were observed for young children. ${ }^{14}$ Viral interference, one virus interfering with another's replication, is a known issue for multivalent live virus vaccine platforms and has been suggested as a cause for the low efficacy and unbalanced immune response. ${ }^{15-17}$ As an alternative to whole-virus platforms, subunit vaccines using the DENV ectodomain (E) antigen are being explored.

Subunit vaccines avoid the viral interference issues associated with whole virus vaccines and have an improved safety profile, allowing vaccination of young, old, and immunocompromised populations. ${ }^{18}$ However, subunit based approaches also tend to have limited immunogenicity, requiring adjuvants or particle delivery platforms to boost immunogenicity and induce longterm immunity. ${ }^{19}$ Particulate subunit vaccine platforms combine an antigen with a nanoparticle to improve the biodistribution, cell uptake, and overall immunogenicity. ${ }^{20,21}$ DENV $E$ has been the main target for subunit vaccine development. Previous work showed the immunogenicity of $\mathrm{E}$ proteins can be improved by adsorbing them to nanoparticle surfaces. Both the magnitude and the neutralization efficiency of the induced DENV-specific IgG responses was significantly higher in the particulate antigen groups. ${ }^{22,23}$ While these formulations improve the strength of the immune response, adsorbed formulations are not known to assemble DENV-E into the complex quaternary structure seen on the surface of DENV. These complex structures have been strongly linked to highly neutralizing and serotype-specific epitopes. ${ }^{24-26}$ To achieve the assembly, display, and characterization of these native $\mathrm{E}$ protein structures, $\mathrm{E}$ must be covalently attached to a particulate platform. Currently, we are aware of only one particulate platform (nanotubes of high aspect ratios) reported to conjugate and deliver recombinant DENV $3 \mathrm{E}$ protein in vivo. 27

The goal of this work is to develop and test a surface conjugated DENV E particulate platform that can be expanded upon in future work. EDC/NHS chemistry was chosen for these conjugations because it is a common biochemical technique that uses aqueous conditions and can target either of the two chemical groups (amines and carboxylic acids) naturally found in DENV E. ${ }^{28}$ EDC/NHS reactions involve an activation step, in which carboxylic acid is activated by EDC then NHS, followed by a conjugation step in which a nucleophile, such as an amine, reacts with the activated group to form an amide bind. The NHS intermediate is more stable, but both the EDC and NHS intermediates will quickly hydrolyze in aqueous solutions. ${ }^{29}$ By conjugating DENV $\mathrm{E}$ to cationic (amine) or anionic (carboxylic acid) particles with different functional handles the effects of $\mathrm{E}$ conjugation through either its amine or carboxylic groups can be tested. EDC/NHS chemistry is advantageous because it does not require synthetic engineering of DENV E and can be used to test both naturally available chemical conjugation groups. ${ }^{30,31}$ However, caution should be observed as conjugation using native chemical groups may disturb the structure of DENV E as well as the antigenic epitopes.

Rod-shaped $\left(80 \times 80 \times 320 \mathrm{~nm}^{3}\right)$ polyethylene glycol (PEG) based polymeric particles with controlled surface chemistry were manufactured using particle replication in nonwetting template (PRINT) technology which allows precise control of size, shape, and chemical composition. ${ }^{32-35}$ Amine functional chemical handles that allow for chemical conjugation and give the particles a cationic charge are polymerized into the particle matrix. These amines can be converted to carboxylic acid groups changing the particle surface charge to anionic at physiologically relevant $\mathrm{pH} .{ }^{33}$ While particle size and surface chemistry are important, cationic particles induce stronger cell uptake and activation for an enhanced cell-mediated immune response. In general, anionic particles avoid cell uptake and may traffic directly to the lymph node, allowing for direct interaction of surface displayed antigen with $\mathrm{B}$ cells. ${ }^{21,36-38}$ Anionic PRINT-hydrogel particles drain lymph nodes more effectively than cationic particles after subcutaneous injection, though cationic particles have enhanced cell uptake and overall immune response in pulmonary vaccine applications. ${ }^{30,31,39}$ In this study we have optimized an EDC/NHS conjugation strategy to covalently attach DENV-E to the surface of anionic and cationic nanoparticles. We have analyzed the effects of chemical conjugation on the integrity of the E protein structure and epitope display on the particle surface, as well as the effects of conjugation on the dengue virus-specific immunity in mice.

\section{RESULTS}

Tween-20 Minimizes DENV2 RecE Adsorption while Allowing Conjugation. Using the protocols outlined in 

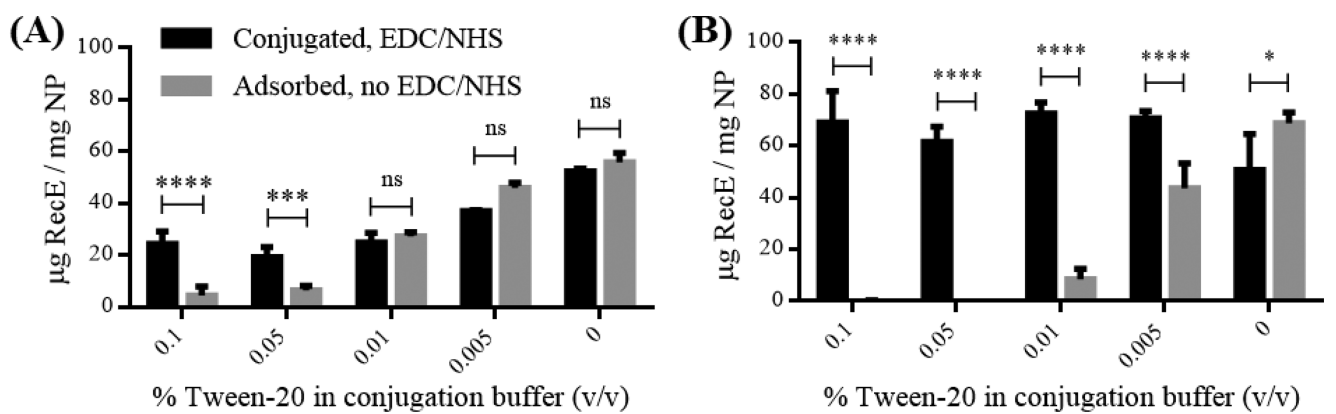

Figure 1. Conjugation and adsorption of RecE to cationic and anionic NP surface. Conjugation results of cationic particles (A) and anionic particles (B) charged with $100 \mu \mathrm{g} \mathrm{RecE} / \mathrm{mg}$ particle either with or without reaction reagents (EDC/NHS) using increasing concentrations of Tween-20 (0, $0.005,0.01,0.05,0.1 \% \mathrm{v} / \mathrm{v})$ in the $\mathrm{pH} 7.2$ conjugation buffer. Following protocol in Scheme 1. Protein mass on the particle surface $(y$-axis) was analyzed by BCA assay after 3 washes in PBS. Statistics: two-way ANOVA with Tukey's test, $*, p<0.05, * * *, p<0.001, * * * *, p<0.0001$.

Scheme 1, the ability of Tween-20 to prevent nonspecific RecE adsorption, while allowing EDC/NHS conjugation, was tested (Figure 1). Groups without EDC/NHS were used to test for background adsorption during the conjugation. For cationic particles, the adsorption background was minimized to $1-3 \mu \mathrm{g}$ $\mathrm{RecE} / \mathrm{mg} \mathrm{NP}$ at $0.1 \%$ Tween-20, while RecE adsorption to anionic particles was negligible at Tween-20 concentrations above $0.05 \%$. Conjugation levels of RecE decreased as Tween20 concentration increased from 0 to $0.01 \%$, and stabilized at concentrations of 0.05 and $0.1 \%$. Conjugations were more efficient on anionic particles and adsorption levels were minimal for both particle charges at Tween-20 concentrations above $0.05 \%$. Various buffers of high salinity, high or low $\mathrm{pH}$, or various detergents were unable to remove RecE adsorbed to particles when Tween-20 was not included in the reaction buffer (Figure S1).

Particulate formulations were characterized for surface conjugated RecE ( $\mu \mathrm{g}$ RecE/mg NP), particle size by DLS (Table 1) and SEM (Figure S2), and surface charge (Table 1). The amount of conjugated RecE protein was slightly lower in the groups conjugated in $0.1 \%$ Tween-20 reaction buffer, designated as NP-RecE T (Table 1). Particle size and surface

Table 1. NP-RecE Characterization: Size, Surface Charge, and Protein Loading RecE Was Conjugated to Cationic (+) or Anionic (-) Particle Groups with Either 0\% (NP-RecE) or $0.1 \%$ Tween-20 (NP-RecE T) in the Conjugation Buffer ${ }^{a}$

$\begin{array}{ccccr}\begin{array}{c}\text { sample } \\ \text { name }\end{array} & \begin{array}{c}\text { protein density } \\ (\mu \mathrm{g} \mathrm{RecE} / \mathrm{mg} \\ \mathrm{NP})\end{array} & \begin{array}{c}\text { hydrodynamic } \\ \text { diameter }(\mathrm{nm})\end{array} & \begin{array}{c}\text { PDI } \\ \text { (polydispersity } \\ \text { index })\end{array} & \begin{array}{r}\text { surface } \\ \text { charge }\end{array} \\ \begin{array}{c}(+) \mathrm{NP}- \\ \text { RecE T }\end{array} & 39.3 & 275 & 0.197 & 36.4 \\ \begin{array}{c}(+) \mathrm{NP}- \\ \text { RecE }\end{array} & 61.3 & 276 & 0.159 & 35.7 \\ \begin{array}{c}(+) \mathrm{NP} \\ \text { Blank }\end{array} & 0.0 & 270 & 0.151 & 34.7 \\ \begin{array}{c}(-) \mathrm{NP}- \\ \begin{array}{c}\text { RecE T } \\ (-) \mathrm{NP}-\end{array}\end{array} & 36.0 & 262 & 0.185 & -35.1 \\ \begin{array}{c}\text { RecE } \\ (-) \mathrm{NP}\end{array} & 49.1 & 247 & 0.117 & -34.0 \\ \text { Blank } & 0.0 & 276 & 0.150 & -37.1 \\ & & & & \end{array}$

${ }^{a}$ Blank particles of each charge are included for comparison. Protein density was obtained by measuring particulate mass using a TGA and protein mass on the particle surface using a BCA assay with a particle background subtracted. The hydrodynamic particulate diameter, PDI, and surface zeta potential were determined by dynamic light scattering (DLS). charge were determined by dynamic light scattering. Both $(+)$ as (-) NP-RecE groups showed minimal change in size or charge compared to their respective NP-Blank controls. All formulations display low PDI values, indicating a monodisperse particle distribution and that protein conjugation did not cause particle aggregation.

(-) NP-RecE T Maintains RecE Structure after Conjugation. The intrinsic fluorescence of sRecE was used to evaluate protein structural stability in particulate groups (Figure 2A). A drop in the intrinsic fluorescence, and/or a shift of the peak to longer wavelengths, indicates a change to the tertiary structure of a protein, and is often observed in proteins undergoing denaturation or misfolding as a result of the aromatic amino acids (tryptophan, tyrosine, and phenylalanine) being exposed to increasingly hydrophilic environments. ${ }^{40}$ Figure S3 displays the relative locations of the aromatic amino acids in RecE in comparison to the DENV2 epitopes tested, showing a strong presence in the 3H5 and 3F9 epitope regions and little presence in the 4G2 or $1 \mathrm{C} 19$ region. Conjugating RecE to anionic (-) particles did not affect the intrinsic fluorescence compared to a control of sRecE mixed with anionic particles immediately before the time of the reading. This control accounts for the fluorescence absorption of the PRINT polymeric PEG particles, as shown in Figure S4. ${ }^{41,42}$ RecE conjugated to cationic (+) particles had a lower intrinsic fluorescence curve than sRecE mixed with cationic particles, which had a similar reading to the anionic particulate groups. Intrinsic fluorescence data in additional testing, comparing EDC/NHS treated and untreated sRecE, suggests that the diminished signal is due to EDC/NHS treatment (Figure S5). However, EDC/NHS treatment did not diminish epitope display in sRecE. It is inconclusive whether the drop in intrinsic fluorescence of the cationic conjugated formulations is caused solely by the EDC/NHS treatment or is a combination of EDC/NHS treatment and sRecE conjugation through its native carboxylic acid groups.

A capture ELISA was used to analyze the effect of conjugation on RecE epitope display. Serially diluted soluble and particulate RecE groups were captured through a Cterminal 6xHis tag on $\mathrm{Ni}^{2+}$-coated ELISA plates (Figure 2B). Analysis included four dengue-specific monoclonal antibodies (4G2, 3H5, 1C19, and 3F9) and two polyclonal sera (DT000 and DT001) (Table 2). RecE conjugated to anionic particles in the presence of Tween-20 shows signals comparable to untreated sRecE. The absence of Tween-20 during the conjugation lowered the overall signals, but the relative signals between $\mathrm{mAbs}$ does not change. As indicated by the intrinsic 
(A)
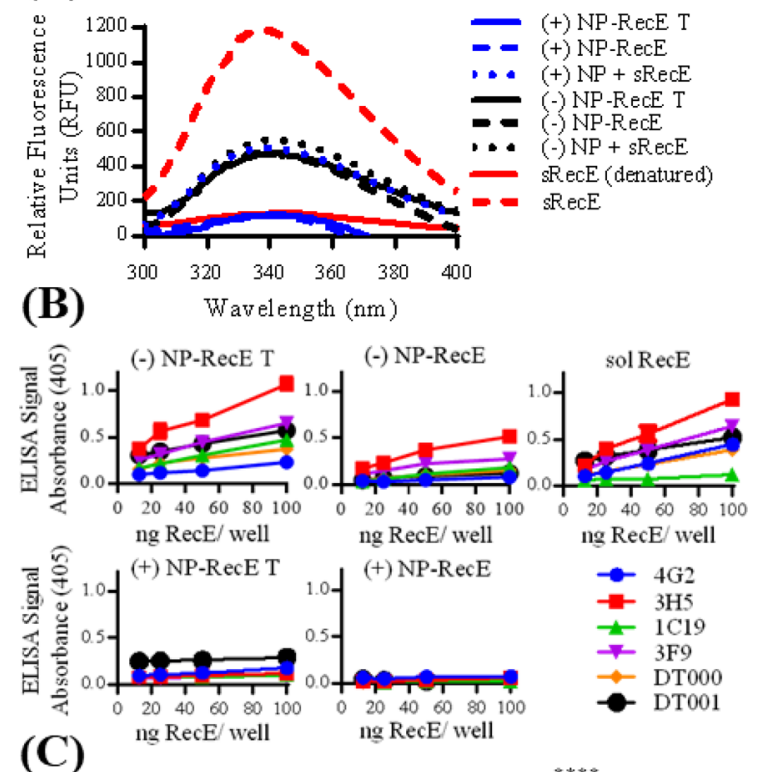

(C)

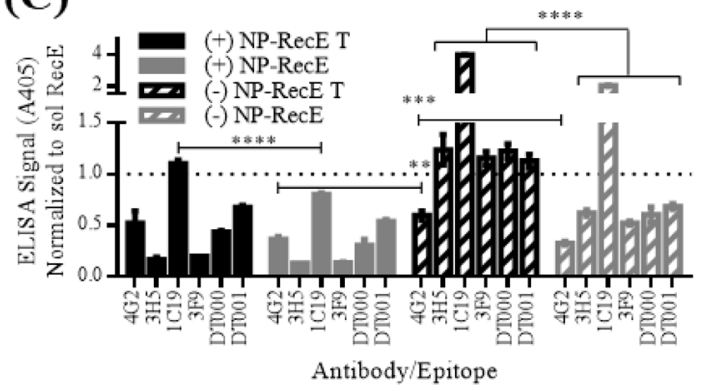

Figure 2. Characterization of RecE in NP-RecE formulations. (A) Intrinsic fluorescence readings of sRecE, NP-RecE groups, and blank particles in solution with sRecE as a controls for the fluorescence absorption of the particles. $\operatorname{secE}$ (denatured) sample was prepared by boiling at $90{ }^{\circ} \mathrm{C}$ for $1 \mathrm{~h}$. Blank particle or nonparticle solutions were subtracted as background. Fluorescence emission spectra are displayed from 300 to $400 \mathrm{~nm}$ with a $280 \mathrm{~nm}$ excitation wavelength. (B) ELISA signals for 4 monoclonal antibodies and 2 polyclonal sera readings dosed to wells by RecE amount at $12.5,25,50$, and $100 \mathrm{ng} /$ ELISA well. (C) ELISA results for each particulate formulation at $100 \mathrm{ng} /$ well, normalized to the sRecE signal. The dotted line at 1 is equivalent to $\mathrm{sRecE}$ signal. Intrinsic fluorescence and ELISA analysis were done with samples within a day of conjugation. (-) NP-RecE T signals (other than 4G2) significant compared to all other groups. Statistics: two-way ANOVA with Tukey's test, $*, p=<0.05$. ***, $p<0.001$, $* * * *, p<0.0001$.

fluorescence data conjugation to cationic particles has a major impact on the epitope display or availability of RecE. The conjugation effects on RecE display is exemplified by normalizing antibody binding signals of particulate RecE to sRecE, where epitope display is $<1,>1$, or equal to 1 and consequently that of soluble RecE (Figure $2 \mathrm{C}$ ). Though the intrinsic fluorescence data showed little difference between the overall tertiary structure of the anionic formulations, the inclusion of Tween-20 during conjugation increased the signal of all the epitopes tested. The difference in results between these two techniques speaks to the precision and fragility of the dengue E protein's structure in certain B cell epitopes. Overall, the data shows that the anionic particle groups have better epitope display than the cationic particle groups and that the presence of Tween is advantageous for epitope availability.
Table 2. Antibodies and Polyclonal Sera Used in Analysis ${ }^{a}$

\begin{tabular}{|c|c|c|c|c|c|}
\hline $\mathrm{mAb}$ & $\mathrm{M} / \mathrm{H}$ & serotype & $\begin{array}{c}\text { neutralization } \\
(\mathrm{W} / \mathrm{M} / \mathrm{S})\end{array}$ & binding region & ref \\
\hline $4 \mathrm{G} 2$ & M & All & W & $\begin{array}{l}\text { DII Fusion } \\
\text { Loop }\end{array}$ & 55 \\
\hline $3 \mathrm{H} 5$ & M & DV2 & $S(\mathrm{DV} 2)$ & $\begin{array}{l}\text { DIII Lateral } \\
\text { Ridge }\end{array}$ & 56 \\
\hline $1 \mathrm{C} 19$ & $\mathrm{H}$ & All & $\begin{array}{l}\mathrm{S}(\mathrm{DV} 1-3), \mathrm{M} \\
(\mathrm{DV} 4)\end{array}$ & DII BC Loop & 57 \\
\hline $3 \mathrm{~F} 9$ & $\mathrm{H}$ & DV2 & S (DV2) & EDIII & 58 \\
\hline DT000* & $\mathrm{H}$ & All & $\begin{array}{l}\mathrm{S}(\mathrm{DV} 1-3), \mathrm{M} \\
(\mathrm{DV} 4)\end{array}$ & N/A & 59 \\
\hline DT001* & $\mathrm{H}$ & DV2 & $\begin{array}{c}\text { S (DV2), W } \\
(\text { DV1,3,4) }\end{array}$ & N/A & 59 \\
\hline
\end{tabular}

${ }^{a}$ A panel of four mouse or human $(\mathrm{M} / \mathrm{H})$ derived monoclonal antibodies and 2 polyclonal patient sera samples $(*)$ were used to characterize RecE. The dengue serotype reactivity, neutralization strength (Weak/Moderate/Strong), and binding region are noted.

(-) NP-RecE T Elicits IgG and Virus Neutralizing Titers. In order to determine the immunogenicity of particulate RecE in the different NP formulations, mice were immunized subcutaneously with $5 \mu \mathrm{g}$ RecE either soluble or in a particulate formulation. Immunizations were given on days 0,21 , and 63 and blood draws were taken on days $0,21,28,56,70$, and 112 (Figure 3A). DENV2 specific IgG end point dilution (EPD) titers 112 days post immunization were determined (Figure 3B). The anionic formulations conjugated in the presence of Tween-20 induced higher IgG titers than soluble RecE in terms of mean value, although statistical analysis does not show a significant difference $(p=0.14)$. Additionally, the IgG titer of the Tween-20 anionic formulation was above that of its cationic and non-Tween anionic particulate counterparts. These results are in line with the protein structure and epitope display in the particulate groups, especially the cationic formulations.

DENV 2 specific virus neutralization titers were determined for day 28, 56, 70, and 112 using a Vero cell based assay and are expressed as Neut $_{50}$ values (Figure 3C). The overall neutralization titers of the anionic particulate groups, specifically the anionic Tween-20 formulation, increased over time until day 70 and decreased slightly after. In line with the lower IgG titers, cationic nanoparticle formulations did not induce potent neutralizing antibody titers. RecE conjugated to anionic particles in the presence of Tween-20 induced neutralizing antibody titers similar to sRecE and sRecE + alum.

PBST Maintains RecE Structure in Storage Better than PBS. The nanoparticle formulation used for booster immunizations were all prepared at day 0 and subsequently stored at $4{ }^{\circ} \mathrm{C}$ in PBS. To analyze the effect of storage time on epitope display and stability, conjugated nanoparticles were stored for 3 weeks in PBS with or without Tween-20 (0.05\% v/v). The mAb and polyclonal sera binding signal to anionic nanoparticles was normalized against the binding signal to soluble RecE (Figure $4 \mathrm{~A})$. Other than $1 \mathrm{C} 19$, each $\mathrm{mAb}$ and polyclonal sera showed decreased binding over time to samples stored in PBS. When samples were stored in PBST only 3F9 and DT000 binding decreased in binding efficiency. 1C19 and DT001 binding increased in the PBST group. 3H5, 3F9, and DT000 also displayed stronger signals on day 1 in the groups that had been washed and stored in PBST after the conjugation reaction. Binding ratios of day 1 and day 21 were analyzed for the two storage conditions where a ratio of 1 would indicate no change in epitope display, $<1$ would suggest a decrease, and $>1$ an increase (Figure 4B). As with the addition of Tween-20 to the 
(A)

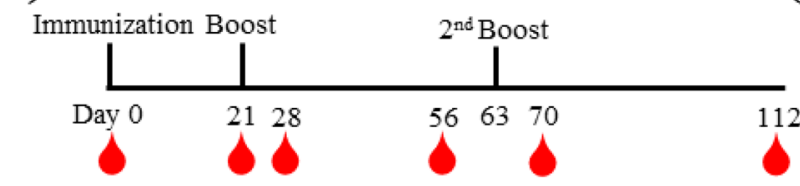

(B)

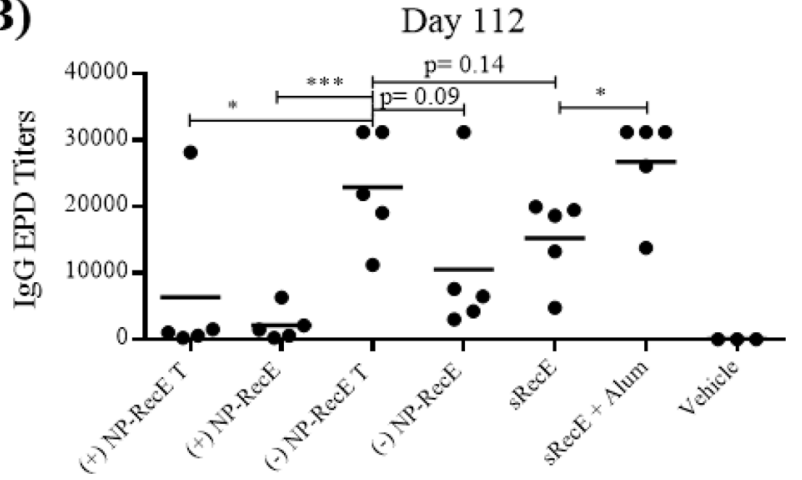

(C)
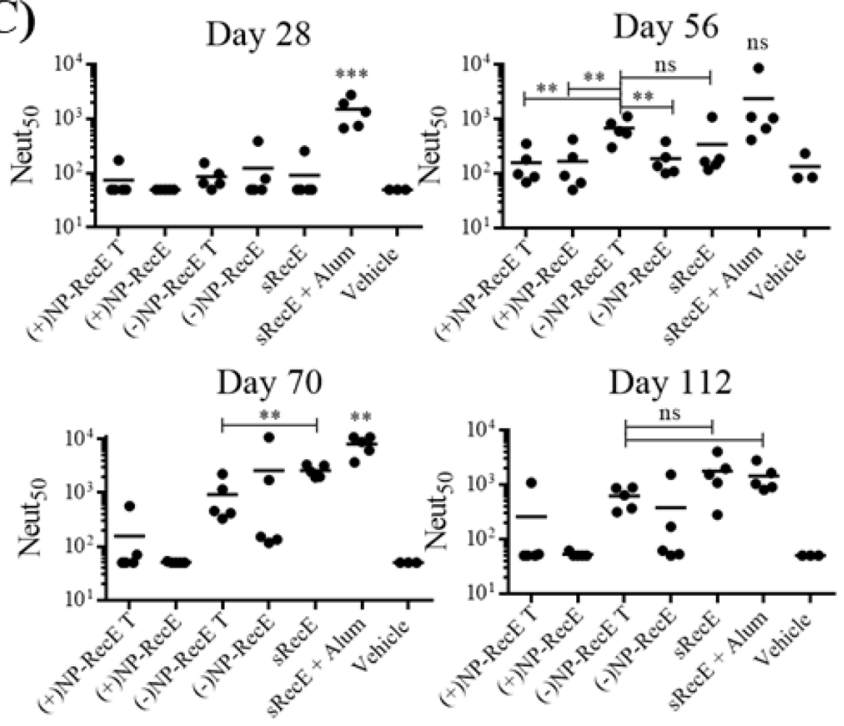

Figure 3. Immune response to NP-RecE formulations. BALB/C mice were immunized subcutaneously with $5 \mu \mathrm{g}$ RecE soluble, particulate, or alum adjuvanted soluble RecE groups according to the (A) timeline. (B) IgG in day 112 sera captured with inactivated DENV2 virus. (C) Virus neutralization. Particulate samples were made before the first injection and stored in PBS at $4{ }^{\circ} \mathrm{C}$ for the duration of the experiment. Statistics: unpaired two-tail $t$ test, $*, p<0.05$, **, $p<0.01$, ***, $p<0.001$.

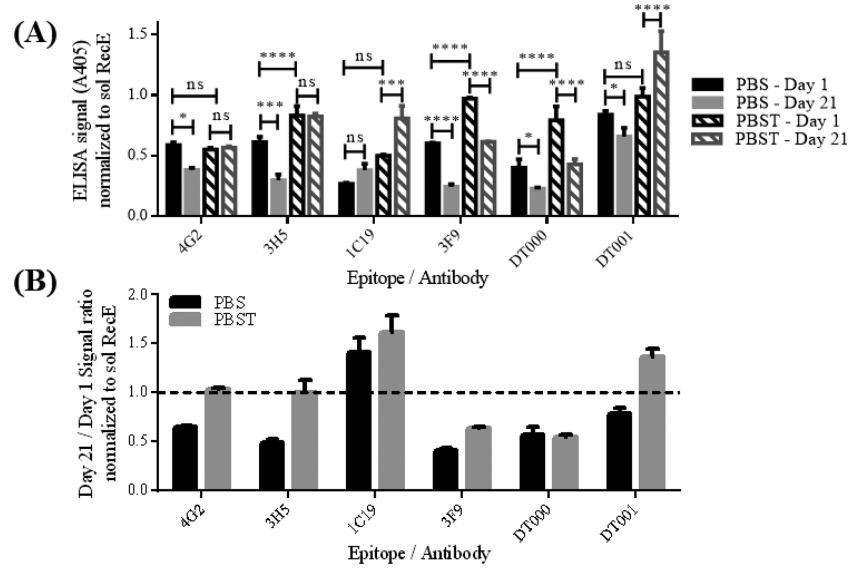

Figure 4. Structural analysis of NP-RecE during storage. (A) Structural analysis of RecE in (-)NP-RecE $\mathrm{T}$ formulations washed and stored in either PBS or PBST buffer, on days 1 and 21 of storage in either PBS or PBST storage buffer. (B) Ratio of normalized epitope signals on day 21 to day 1 for NP-RecE stored in PBS/PBST. Statistics: two-way ANOVA with Tukey's test, $*, p<0.05, * * *, p<0.001$, ****, $p<$ 0.0001 .

conjugation buffer, Tween-20 in the storage buffer improved and maintained epitope signals.

\section{DISCUSSION}

Our data indicates that adding Tween-20 to the reaction buffer in covalently conjugated RecE particulate formulations not only minimizes adsorption, but additionally improves protein stability translating to a more DENV-specific and neutralizing immune response. A linear increase of Tween-20 concentration in the reaction buffer decreases antigen-specific adsorption to the particle surface during conjugation. The presence of Tween-20 during the reaction translates to an improved epitope display which additionally induces a stronger IgG response. The decreasing $\mathrm{mAb}$ and serum binding signals over time of RecE nanoparticle formulations stored in the absence of
Tween-20 indicates the importance of the detergent for maintaining structural epitopes that are important for inducing DENV-specific and neutralizing antibody responses. The epitope enhancing effects of Tween-20 are likely caused by the prevention of protein interactions with the nanoparticles, which often cause background in adsorption, leading to changes in the protein structure. Protein interactions with surfaces, especially nanoparticles, are well documented to cause protein denaturation and unfolding over time, as the protein adsorbs, often changing protein structure to form new and sometimes cryptic epitopes. ${ }^{37,43-46}$ Additionally, adsorbed protein can be very stable, which may explain why RecE could not be "washed off” of particles using buffers with high salinity, high or low $\mathrm{pH}$, or concentrated detergents (Figure S1). ${ }^{47}$ Other recent studies have also found that detergents can be used to minimize protein adsorption to nanoparticles. ${ }^{48}$ Future experiments should use the epitope friendly storage conditions shown here to preserve RecE structure and induce a more potent neutralizing response in vivo.

Decreased antibody binding and moderate induction of IgG and neutralizing titers do not exclude cationic particles from further development. Additional testing indicated that the use of EDC/NHS chemistry for conjugations may have damaged the $\mathrm{E}$ protein antigen (Figure S5). Therefore, other conjugation methods may yield improved epitope display in cationic particle formulations. It is also possible that after the conjugation, the close proximity of RecE to the cationic particle surface enhances adsorption interactions, which often occur between proteins and particle surfaces. ${ }^{49-51}$ It may be important to explore cationic particle formulations in future work as they have been shown to activate both CD8 and CD4 T cells. ${ }^{23,31,52}$

\section{CONCLUSION}

The particulate formulations here do not provide a significantly stronger immune response compared to soluble RecE. However, they do represent a platform where the display of a DENV E antigen can be controlled while it is covalently attached to the surface of a nanoparticle. The described 
methods improve the stability of RecE and demonstrate a set of assays to characterize conjugated NP-RecE formulations. The correlation between the epitope conservation and the in vivo immunogenicity of NP-RecE formulations allows for fast screening of new nanoparticle based candidate antigens without going through the long in vivo experiments. Recently, it has become clear that the assembly of DENV E into quaternary structures may be paramount to the ability to induce a strongly neutralizing and serotype specific response. ${ }^{24-26}$ Additional work has shown the ability to generate quaternary epitopes from DENV-E subunits via various methods. ${ }^{53,54}$ Metz et al. describes a method to assemble DENV2 E dimers on a solid surface. The assembled DENV2 E dimers are shown to display highly neutralizing quaternary epitopes. The work here describes the first covalently conjugated particulate platform that allows for the display of DENV E antigen and correlates the strength of that display to the immune response. The next step in the development of a particulate DENV E platform will be to combine the methods described here with those to form quaternary epitopes, such as dimers, on other surfaces. If these methods can be readily transferred to a nanoparticle surface to display DENV E in the same structures found on the surface of the DENV virion, it will be a large step toward developing a safe and efficacious DENV E subunit particulate vaccine.

\section{EXPERIMENTAL PROCEDURES}

Cells and Viruses. Vero cells for neutralization assay were maintained as monolayer cell cultures at $37{ }^{\circ} \mathrm{C}$ with $5 \% \mathrm{CO}_{2}$, in DMEM (Gibco) media supplemented with $1 \%$ nonessential amino acids, $100 \mathrm{U} / \mathrm{mL}$ penicillin, $100 \mu \mathrm{g} / \mathrm{mL}$ streptomycin, and $5 \%$ fetal bovine serum (FBS), which was lowered to $2 \%$ during infection. EXPI293-cells were used for the production of soluble recombinant $\mathrm{E}$ (sRecE) and were maintained in suspension in EXPI293 Expression Medium (Life Technologies). Cultures were passaged to $3 \times 10^{5}$ cells $/ \mathrm{mL}$ when cell densities of $3.5 \times 10^{6}$ cells $/ \mathrm{mL}$ were reached. DENV2 S-16803 was used to determine antibody titers and in neutralization assays.

Recombinant E Protein Production and Purification. RecE proteins were produced and purified as previously described. $^{22}$ RecE (aa1-395) was expressed with a c-terminal 6X histidine in the EXPI293 transient expression system (Fisher) using manufacturer protocols. Supernatants were purified using nickel affinity chromatography with tangential flow filtration. Eluted fractions were pooled and subjected to size exclusion chromatography and subsequently concentrated in PBS with $10 \%(\mathrm{v} / \mathrm{v})$ glycerol. Final fractions were pooled, flash frozen in liquid nitrogen, and stored at $-80{ }^{\circ} \mathrm{C}$.

Particle Fabrication and Characterization. Particles were produced using PRINT technology as described previously. ${ }^{30-35}$ The particles are composed of $69 \%$ tetra(ethylene glycol) monacrylate (monomer), 20\% aminoethyl methacrylate (chemical handle), 10\% M.W. 700 polyethyelene glycol diacrylate (cross-linker), 1\% diphenyl(2,4,6-trimethylbenzoyl) phosphine oxide (photo initiator) (Table S1). All particle components were purchased from Sigma. Particles were produced using $80 \times 80 \times 320 \mathrm{~nm}$ rod-shaped molds provided by Liquidia. Particle size, shape, and zeta potential were characterized by scanning electron microscopy (SEM) and dynamic light scattering (DLS) using a Malvern Nanosizer ZS with adjusted parameters for polyethylene glycol (PEG). Particle mass concentration was determined using thermogravi- metric analysis on a thermogravimetric analyzer (TGA) from TA Instruments.

Particle Conversion from Cationic to Anionic. One milligram of cationic particle were washed into dimethylformamide (DMF) (Fisher) and subsequently mixed with $15 \mathrm{mg}$ succinic anhydride (Sigma), $10 \mu \mathrm{L}$ pyridine in $1 \mathrm{~mL}$ total solvent for $30 \mathrm{~min}$. After conversion, the particles were washed twice with DMF and 3 times in water. Anionic charge was confirmed by DLS.

Protein Quantification by BCA Assay. Protein concentration on particle surfaces was determined by using a bicinchoninic acid assay (BCA) kit (Fisher) following manufacturers protocol.

RecE Conjugation to Particles Using EDC/NHS Chemistry. To conjugate sRecE to cationic nanoparticles, sRecE was activated with EDC (Sigma) at a $1000 \times$ molar ratio and NHS (Fisher) at a $5000 \times$ molar ratio for $15 \mathrm{~min}$ in $0.05 \mathrm{M}$ 2-(N-morpholino) ethanesulfonic acid (Fisher) buffer at $\mathrm{pH} 6.0$ while shaking at $1400 \mathrm{rpm}$. An equal volume of cationic particles were added to the reaction in $0.1 \mathrm{M}$ sodium phosphate (Sigma) buffer at $\mathrm{pH} 7.2$. After $2 \mathrm{~h}$ on a shaker at $1400 \mathrm{rpm}$, the NP-RecE were washed twice with $2 \times$ PBS (Corning) and 1 time with PBS.

RecE was conjugated to anionic particles by activating the anionic particles with EDC and NHS in a $1000 \times$ molar ratio to sRecE in MES buffer ( $\mathrm{pH}$ 6). RecE was added to $0.1 \mathrm{M}$ sodium phosphate buffer for $2 \mathrm{~h}$ after which the particles were washed 2 times with $2 \times$ PBS and 1 time with PBS.

$\mathrm{RecE}$ adsorption during the conjugation protocol was evaluated by performing the protocol without adding EDC/ NHS with Tween-20 (Fisher) in the reaction buffer at varying concentrations. Samples were washed 3 times with $1 \mathrm{~mL}$ of PBS before protein quantification with BCA assay.

Intrinsic Fluorescence Measurements. The intrinsic fluorescence of covalently bound particulate RecE (NPRecE), soluble RecE (sRecE), or soluble RecE mixed with particles just before readings (NP blank + sRecE) was measured using top read fluorescence with excitation at 280 $\mathrm{nm}$ and absorbance from 300 to $400 \mathrm{~nm}$ by steps of $1 \mathrm{~nm}$ Spectra Max microplate reader (Molecular Devices) in 96 well black opaque plates (Nunc, Thermo). Each sample was dosed with $10 \mu \mathrm{g} \operatorname{RecE}(2 \mu \mathrm{M})$ and $100 \mu \mathrm{g} \mathrm{NP}$ in the appropriate samples.

ELISA. $\mathrm{Ni}^{2+}$-coated plates (Pierce) were loaded with 100, 50, 25 , or $12.5 \mathrm{ng} \mathrm{RecE} /$ well of either NP-RecE or sRecE formulations in TBS buffer (50 mMTris, $150 \mathrm{mMNaCl}, \mathrm{pH}$ 7.5) for $1 \mathrm{~h}$ at $37{ }^{\circ} \mathrm{C}$. The plates were washed (TBS+0.05\% Tween-20) and blocked (washing buffer $+3 \%$ skim milk) for 1 $\mathrm{h}$ at $37^{\circ} \mathrm{C}$. The blocking buffer was discarded and the plates were incubated for $1 \mathrm{~h}$ at $37{ }^{\circ} \mathrm{C}$ with $100 \mathrm{ng} /$ well of 4G2, 3H5, 3F9, and 1C19 monoclonal antibodies (mAbs) or 1:10 diluted human derived serum of DENV infected individuals DT000 and DT001 (Table 2). Next, plates were washed and treated with alkaline phosphatase (AP) conjugated $\alpha$-human IgG (Sigma, 1:2500) or $\alpha$-mouse IgG (Sigma, 1:1000) in blocking buffer for $45 \mathrm{~min}$ at $37{ }^{\circ} \mathrm{C}$. Plates were subsequently washed and developed using AP-substrate (Sigma). Absorbance was measured at $405 \mathrm{~nm}$.

Mouse Immunizations. Female $\mathrm{Balb} / \mathrm{c}$ mice were purchased from Jackson Laboratory and immunized at age 6-12 weeks. Mice were subcutaneously immunized in the flank with sRecE or NP-sRecE formulations dosed at $5 \mu \mathrm{g}$ RecE per mouse per dose, in $300 \mu \mathrm{L}$ volume containing 9.25 wt \% 
sucrose. Mice were primed on day 0 and boosted on days 21 and 63 post prime. Serum samples were collected by submandibular bleeding on days $21,28,57,70$, and 112. All experiments involving mice were carried out in accordance with an animal use protocol approved by the University of North Carolina Animal Care and Use Committee.

Evaluation of Vaccine Induced Antibodies by IgG End Point Dilution Assay. ELISA plates were coated with $100 \mathrm{ng} /$ well of IM7 mAb in $50 \mathrm{mM}$ carbonate/bicarbonate buffer $\mathrm{pH}$ 9.6 and incubated overnight at $4{ }^{\circ} \mathrm{C}$. The next day, the plates were washed in washing buffer (PBS with $0.05 \%$ Tween-20) and blocked with blocking buffer (washing buffer $+3 \%$ skim milk) for $1 \mathrm{~h}$ at $37^{\circ} \mathrm{C}$. Next, plates were loaded with DENV2 in blocking buffer and incubated for $1 \mathrm{~h}$ at $37^{\circ} \mathrm{C}$. After washing, the immunized mice sera was serially diluted in blocking buffer and loaded on the plates for $1 \mathrm{~h}$ at $37{ }^{\circ} \mathrm{C}$. The plates were washed and subjected to AP-conjugated $\alpha$-mouse IgG (Sigma, 1:1000) for $45 \mathrm{~min}$ at $37^{\circ} \mathrm{C}$ and developed after washing with AP-substrate (Sigma). Absorbance was measured at $405 \mathrm{~nm}$. The vehicle group was used to determine the background signal of the assay. The dilution where the sera from the experimental groups reaches background levels was calculated using GraphPad Prism software and used as a measure of end point dilution titer.

Evaluation of Vaccine Induced Antibodies by DENV2 Neutralization Assay. A flow cytometry based neutralization assay was used to measure DENV neutralizing antibodies, which has been previously described in detail. ${ }^{33}$ Vero cells were seeded in a 96-well culture plate at $2.5 \times 10^{4}$ cells/well and incubated $24 \mathrm{~h}$ at $37{ }^{\circ} \mathrm{C}$. Mice sera were serially diluted and incubated for $45 \mathrm{~min}$ with appropriate amount of virus to establish a $\sim 15 \%$ infection (determined previously). Next, the virus/sera solution was added to the cells. The next day, cells were trypsinized and fixed in $4 \%$ paraformaldehyde. Next, cells were spun down and blocked and incubated with Alexa Fluor 488 conjugated anti-prM Mab 2H2. The percentage of infected cells was determined by flow cytometry using a Guava Flow Cytometer (EMD Millipore) and the neutralizing efficiency of the sera was expressed as neut50 values (the dilution where $50 \%$ of the virus was neutralized) calculated using GraphPad Prism software.

\section{ASSOCIATED CONTENT}

\section{S Supporting Information}

The Supporting Information is available free of charge on the ACS Publications website at DOI: 10.1021/acs.bioconjchem.8b00090.

RecE adsorption and particle formulation; SEM; RecE structure; light absorbance of PRINT PEG particles; Characterization of EDC/NHS activated sRecE (PDF)

\section{AUTHOR INFORMATION}

\section{Corresponding Author}

*E-mail: smtian@email.unc.edu.

\section{ORCID}

Jason E. Coffman: 0000-0002-0418-1166

\section{Notes}

The authors declare the following competing financial interest(s): Joseph M. DeSimone holds a financial stake in Liquidia Technologies.

\section{ACKNOWLEDGMENTS}

This work was financially supported by the National Institute of Health (NIH, AI109784). The authors also thank our industrial partner Liquidia Technology for supplies and support.

\section{REFERENCES}

(1) Bhatt, S., Gething, P. W., Brady, O. J., Messina, J. P., Farlow, A. W., Moyes, C. L., Drake, J. M., Brownstein, J. S., Hoen, A. G., Sankoh, O., et al. (2013) The Global Distribution and Burden of Dengue. Nature 496, 504-507.

(2) Guzman, M. G., Halstead, S. B., Artsob, H., Buchy, P., Farrar, J., Gubler, D. J., Hunsperger, E., Kroeger, A., Margolis, H. S., Martínez, E., et al. (2010) Dengue: A Continuing Global Threat. Nat. Rev. Microbiol. 8, S7-16.

(3) Halstead, S. (1988) Pathogenesis of Dengue: Challenges to Molecular Biology. Science (Washington, DC, U. S.) 239, 476-481.

(4) Siraj, A. S., Oidtman, R. J., Huber, J. H., Kraemer, M. U. G., Brady, J., Johansson, M. A., and Perkins, T. A. (2017) Temperature Modulates Dengue Virus Epidemic Growth Rates through Its Effects on Reproduction Numbers and Generation Intervals. PLoS Neglected Trop. Dis. 11, e0005797.

(5) Gubler, D. J., Reiter, P., Ebi, K. L., Yap, W., Nasci, R., and Patz, J. a. (2001) Climate Variability and Change in the United States: Potential Impacts on Vector- and Rodent-Borne Diseases. Environ. Health Perspect. 109 (Suppl), 223-233.

(6) Hales, S., de Wet, N., Maindonald, J., and Woodward, A. (2002) Potential Effect of Population and Climate Changes on Global Distribution of Dengue Fever: An Empirical Model. Lancet 360, 830834.

(7) Dejnirattisai, W., Jumnainsong, A., Onsirisakul, N., Fitton, P., Vasanawathana, S., Limpitikul, W., Puttikhunt, C., Edwards, C., Duangchinda, T., Supasa, S., et al. (2010) Cross-Reacting Antibodies Enhance Dengue Virus Infection in Humans. Science 328, 745-748.

(8) Halstead, S. B. (2009) Antibodies Determine Virulence in Dengue. Ann. N. Y. Acad. Sci. 1171 (Suppl), E48-56.

(9) Zellweger, R. M., Prestwood, T. R., and Shresta, S. (2010) Enhanced Infection of Liver Sinusoidal Endothelial Cells in a Mouse Model of Antibody-Induced Severe Dengue Disease. Cell Host Microbe $7,128-139$

(10) Vaughn, D. W., Green, S., Kalayanarooj, S., Innis, B. L., Nimmannitya, S., Suntayakorn, S., Endy, T. P., Raengsakulrach, B., Rothman, a L., Ennis, F. a, et al. (2000) Dengue Viremia Titer, Antibody Response Pattern, and Virus Serotype Correlate with Disease Severity. J. Infect. Dis. 181, 2-9.

(11) World Health Organization (2016) Weekly Epidemiological Report; Vol 30, pp 349364; http://www.who.int/wer/2016/wer9130. pdf

(12) Sanofi Pasteur (2016) First Dengue Vaccine Approved in More Than 10 Countries; pp 4-7; https://www.sanofipasteur.com/media/ Project/One-Sanofi-Web/sanofipasteur-com/en/media-room/docs/ P R_20 20161004

FirstDengueVaccineApprovedInMoreThan 10Countries_EN.pdf

(13) Guy, B., Briand, O., Lang, J., Saville, M., and Jackson, N. (2015) Development of the Sanofi Pasteur Tetravalent Dengue Vaccine: One More Step Forward. Vaccine 33, 7100-7111.

(14) Halstead, S. B., and Russell, P. K. (2016) Protective and Immunological Behavior of Chimeric Yellow Fever Dengue Vaccine. Vaccine 34, 1643-1647.

(15) Anderson, K. B., Gibbons, R. V., Edelman, R., Eckels, K. H., Putnak, R. J., Innis, B. L., and Sun, W. (2011) Interference and Facilitation between Dengue Serotypes in a Tetravalent Live Dengue Virus Vaccine Candidate. J. Infect. Dis. 204, 442-450.

(16) Swaminathan, S., Khanna, N., Herring, B., and Mahalingam, S. (2013) Dengue Vaccine Efficacy Trial: Does Interference Cause Failure? Lancet Infect. Dis. 13, 191-192.

(17) Halstead, S. B. (2013) Identifying Protective Dengue Vaccines: Guide to Mastering an Empirical Process. Vaccine 31, 4501-4507. 
(18) Hilleman, M. R. (2000) Vaccines in Historic Evolution and Perspective. J. Hum. Virol. 2, 63.

(19) O'Hagan, D. T., and Valiante, N. M. (2003) Recent Advances in the Discovery and Delivery of Vaccine Adjuvants. Nat. Rev. Drug Discovery 2, 727-735.

(20) De Temmerman, M.-L., Rejman, J., Demeester, J., Irvine, D. J., Gander, B., and De Smedt, S. C. (2011) Particulate Vaccines: On the Quest for Optimal Delivery and Immune Response. Drug Discovery Today 16, 569-582.

(21) Irvine, D. J., Hanson, M. C., Rakhra, K., and Tokatlian, T. (2015) Synthetic Nanoparticles for Vaccines and Immunotherapy. Chem. Rev. 115, 11109-11146.

(22) Metz, S. W., Tian, S., Hoekstra, G., Yi, X., Stone, M., Horvath, K., Miley, M. J., DeSimone, J., Luft, C. J., and de Silva, A. M. (2016) Precisely Molded Nanoparticle Displaying DENV-E Proteins Induces Robust Serotype-Specific Neutralizing Antibody Responses. PLoS Neglected Trop. Dis. 10, 1-17.

(23) Swaminathan, G., Thoryk, E. A., Cox, K. S., Smith, J. S., Wolf, J. J., Gindy, M. E., Casimiro, D. R, and Bett, A. J. (2016) A Tetravalent Sub-Unit Dengue Vaccine Formulated with Ionizable Cationic Lipid Nanoparticle Induces Significant Immune Responses in Rodents and Non-Human Primates. Sci. Rep. 6, 34215.

(24) Dejnirattisai, W., Wongwiwat, W., Supasa, S., Zhang, X., Dai, X., Rouvinsky, A., Jumnainsong, A., Edwards, C., Quyen, N. T., Duangchinda, T., et al. (2014) A New Class of Highly Potent, Broadly Neutralizing Antibodies Isolated from Viremic Patients Infected with Dengue Virus. Nat. Immunol. 16, 170-177.

(25) de Alwis, R., Smith, S. a., Olivarez, N. P., Messer, W. B., Huynh, J. P., Wahala, W. M. P. B., White, L. J., Diamond, M. S., Baric, R. S., Crowe, J. E., et al. (2012) Identification of Human Neutralizing Antibodies That Bind to Complex Epitopes on Dengue Virions. Proc. Natl. Acad. Sci. U. S. A. 109, 7439-7444.

(26) Fibriansah, G., Tan, J. L., Smith, S. A., de Alwis, R., Ng, T., Kostyuchenko, V. A., Jadi, R. S., Kukkaro, P., de Silva, A. M., Crowe, J. E., et al. (2015) A Highly Potent Human Antibody Neutralizes Dengue Virus Serotype 3 by Binding across Three Surface Proteins. Nat. Commun. 6, 6341.

(27) Versiani, A. F., Astigarraga, R. G., Rocha, E. S. O., Barboza, A. P. M., Kroon, E. G., Rachid, M. A., Souza, D. G., Ladeira, L. O., BarbosaStancioli, E. F., Jorio, A., et al. (2017) Multi-Walled Carbon Nanotubes Functionalized with Recombinant Dengue Virus 3 Envelope Proteins Induce Significant and Specific Immune Responses in Mice. J. Nanobiotechnol. 15, 26.

(28) Brinkley, M. (1992) A Brief Survey of Methods for Preparing Protein Conjugates with Dyes, Haptens and Crosslinking Reagents. Bioconjugate Chem. 3, 2-13.

(29) Nakajima, N., and Ikada, Y. (1995) Mechanism of Amide Formation by Carbodiimide for Bioconjugation in Aqueous Media. Bioconjugate Chem. 6, 123-130.

(30) Mueller, S. N., Tian, S., and Desimone, J. M. (2015) Rapid and Persistent Delivery of Antigen by Lymph Node Targeting PRINT Nanoparticle Vaccine Carrier to Promote Humoral Immunity. Mol. Pharmaceutics 12, 1356-1365.

(31) Fromen, C. a, Robbins, G. R., Shen, T. W., Kai, M. P., Ting, J. P. Y., and DeSimone, J. M. (2015) Controlled Analysis of Nanoparticle Charge on Mucosal and Systemic Antibody Responses Following Pulmonary Immunization. Proc. Natl. Acad. Sci. U. S. A. 112, 488-493.

(32) Reuter, K. G., Perry, J. L., Kim, D., Luft, J. C., Liu, R., and DeSimone, J. M. (2015) Targeted PRINT Hydrogels: The Role of Nanoparticle Size and Ligand Density on Cell Association, Biodistribution, and Tumor Accumulation. Nano Lett. 15, 6371-6378.

(33) Perry, J. L., Reuter, K. G., Kai, M. P., Herlihy, K. P., Jones, S. W., Luft, J. C., Napier, M., Bear, J. E., and Desimone, J. M. (2012) PEGylated PRINT Nanoparticles: The Impact of PEG Density on Protein Binding, Macrophage Association, Biodistribution, and Pharmacokinetics. Nano Lett. 12, 5304-5310.

(34) Kapadia, C. H., Tian, S., Perry, J. L., Luft, J. C., and Desimone, J. M. (2016) Reduction Sensitive PEG Hydrogels for Codelivery of
Antigen and Adjuvant to Induce Potent CTLs. Mol. Pharmaceutics 13, 3381-3394.

(35) Rolland, J. P., Maynor, B. W., Euliss, L. E., Exner, A. E., Denison, G. M., and DeSimone, J. M. (2005) Direct Fabrication and Harvesting of Monodisperse, Shape-Specific Nanobiomaterials. J. Am. Chem. Soc. 127, 10096-10100.

(36) He, C., Hu, Y., Yin, L., Tang, C., and Yin, C. (2010) Effects of Particle Size and Surface Charge on Cellular Uptake and Biodistribution of Polymeric Nanoparticles. Biomaterials 31, 36573666.

(37) Nel, A. E., Mädler, L., Velegol, D., Xia, T., Hoek, E. M. V, Somasundaran, P., Klaessig, F., Castranova, V., and Thompson, M. (2009) Understanding Biophysicochemical Interactions at the NanoBio Interface. Nat. Mater. 8, 543-557.

(38) Swartz, M. A., Hubbell, J. A., and Reddy, S. T. (2008) Lymphatic Drainage Function and Its Immunological Implications: From Dendritic Cell Homing to Vaccine Design. Semin. Immunol. 20, $147-156$.

(39) Fromen, C. A., Rahhal, T. B., Robbins, G. R., Kai, M. P., Shen, T. W., Luft, J. C., and DeSimone, J. M. (2016) Nanoparticle Surface Charge Impacts Distribution, Uptake and Lymph Node Trafficking by Pulmonary Antigen-Presenting Cells. Nanomedicine 12, 677-687.

(40) Royer, C. a. (2006) Probing Protein Folding and Conformational Transitions with Fluorescence Probing Protein Folding and Conformational Transitions with Fluorescence. Chem. Rev. 106, 17691784.

(41) Luo, C., Zhang, Y., Zeng, X., Zeng, Y., and Wang, Y. (2005) The Role of Poly(ethylene Glycol) in the Formation of Silver Nanoparticles. J. Colloid Interface Sci. 288, 444-448.

(42) Díaz-Cruz, C., Alonso Nuñez, G., Espinoza-Gómez, H., and Flores-López, L. Z. (2016) Effect of Molecular Weight of PEG or PVA as Reducing-Stabilizing Agent in the Green Synthesis of SilverNanoparticles. Eur. Polym. J. 83, 265-277.

(43) Nel, A. (2006) Toxic Potential of Materials. Science (Washington, DC, U. S.) 311, 622-627.

(44) Kondo, a, Murakami, F., and Higashitani, K. (1992) Circular Dichroism Studies on Conformational Changes in Protein Molecules upon Adsorption on Ultrafine Polystyrene Particles. Biotechnol. Bioeng. 40, 889-894.

(45) Satzer, P., Svec, F., Sekot, G., and Jungbauer, A. (2016) Protein Adsorption onto Nanoparticles Induces Conformational Changes: Particle Size Dependency, Kinetics, and Mechanisms. Eng. Life Sci. 16, 238-246.

(46) Stefani, M. (2008) Protein Folding and Misfolding on Surfaces. Int. J. Mol. Sci. 9, 2515-2542.

(47) Milani, S., Baldelli Bombelli, F., Pitek, A. S., Dawson, K. A., and Rädler, J. (2012) Reversible versus Irreversible Binding of Transferrin to Polystyrene Nanoparticles: Soft and Hard Corona. ACS Nano 6, 2532-2541.

(48) Winzen, S., Schwabacher, J. C., Müller, J., Landfester, K., and Mohr, K. (2016) Small Surfactant Concentration Differences Influence Adsorption of Human Serum Albumin on Polystyrene Nanoparticles. Biomacromolecules 17, 3845-3851.

(49) Dominguez-Medina, S., Kisley, L., Tauzin, L. J., Hoggard, A., Shuang, B., Indrasekara, D. S., Chen, S., Wang, L. Y., Derry, P. J., Liopo, A., et al. (2016) Adsorption and Unfolding of a Single Protein Triggers Nanoparticle Aggregation. ACS Nano 10, 2103-2112.

(50) Billsten, P., Wahlgren, M., Arnebrant, T., McGuire, J., and Elwing, H. (1995) Structural Changes of T4 Lysozyme Adsorption to Silica Nanoparticles Measured by Circular Dichoism. J. Colloid Interface Sci. 175, 77-82.

(51) Pan, H., Qin, M., Meng, W., Cao, Y., and Wang, W. (2012) How Do Proteins Unfold upon Adsorption on Nanoparticle Surfaces? Langmuir 28, 12779-12787.

(52) Nembrini, C., Stano, A., Dane, K. Y., Ballester, M., van der Vlies, A. J., Marsland, B. J., Swartz, M. a, and Hubbell, J. a. (2011) Nanoparticle Conjugation of Antigen Enhances Cytotoxic T-Cell Responses in Pulmonary Vaccination. Proc. Natl. Acad. Sci. U. S. A. 108, E989-97. 
(53) Metz, S. W., Gallichotte, E. N., Brackbill, A., Premkumar, L., Miley, M. J., Baric, R., and de Silva, A. M. (2017) In Vitro Assembly and Stabilization of Dengue and Zika Virus Envelope Protein HomoDimers. Sci. Rep. 7, 4524.

(54) Rouvinski, A., Dejnirattisai, W., Guardado-Calvo, P., Vaney, M.C., Sharma, A., Duquerroy, S., Supasa, P., Wongwiwat, W., Haouz, A., Barba-Spaeth, G., et al. (2017) Covalently Linked Dengue Virus Envelope Glycoprotein Dimers Reduce Exposure of the Immunodominant Fusion Loop Epitope. Nat. Commun. 8, 15411.

(55) Henchal, E. A., Mccown, J. M., Burke, D. S., Seguin, M. C., and Brandt, W. E. (1985) Epitopic Analysis of Antigenic Determinants on the Surface of Dengue-2 Virions Using Monoclonal Antibodies*. Am. J. Trop. Med. Hyg. 34, 162-169.

(56) Pitcher, T. J., Sarathy, V. V., Kiyohiko, M., Gromowski, G. D., Huang, C. Y. H., and Barrett, A. D. T. (2015) Functional Analysis of Dengue Virus (DENV) Type 2 Envelope Protein Domain 3 TypeSpecific and DENV Complex-Reactive Critical Epitope Residues. J. Gen. Virol. 96, 288-293.

(57) Smith, S. A., de Alwis, A. R., Kose, N., Harris, E., Ibarra, K. D., Kahle, K. M., Pfaf, J. M., Xiang, X., Doranz, B. J., de Silva, A. M., et al. (2013) The Potent and Broadly Neutralizing Human Dengue VirusSpecific Monoclonal Antibody 1C19 Reveals a Unique Cross-Reactive Epitope on the Bc Loop of Domain II of the Envelope Protein. mBio 4, e00873-13.

(58) Smith, S. A., de Alwis, A. R., Kose, N., Jadi, R. S., de Silva, A. M., and Crowe, J. E. (2014) Isolation of Dengue Virus-Specific Memory B Cells with Live Virus Antigen from Human Subjects Following Natural Infection Reveals the Presence of Diverse Novel Functional Groups of Antibody Clones. J. Virol. 88, 12233-12241.

(59) Patel, B., Longo, P., Miley, M. J., Montoya, M., Harris, E., and de Silva, A. M. (2017) Dissecting the Human Serum Antibody Response to Secondary Dengue Virus Infections. PLoS Neglected Trop. Dis. 11, $\mathrm{e} 0005554$. 